\title{
Verify Safe Use Conditions
}

National Cancer Institute

\section{Source}

National Cancer Institute. Verify Safe Use Conditions. NCI Thesaurus. Code C128515.

The healthcare provider or setting must verify that required safe use conditions are in place before the drug is dispensed using established processes and procedures. For purposes of this definition, "safe use conditions" refers to any activity that must be completed before the drug is dispensed (e.g., enrollment or completion of a lab test). 